\title{
Surface Relaxation Processes of Poly(methyl methacrylate) Brushes Prepared by Atom Transfer Radical Polymerization
}

\author{
Keiji Tanaka, Ken Kojio, Reiko Kimura, Atsushi Takahara, ${ }^{\dagger}$ and Tisato KajiYama ${ }^{\dagger \dagger}$ \\ Department of Applied Chemistry, Faculty of Engineering, Kyushu University, \\ Fukuoka 812-8581, Japan
}

(Received July 1, 2002; Accepted October 29, 2002)

\begin{abstract}
Well-defined poly(methyl methacrylate) (PMMA) brush layers were prepared onto silicon wafers by surface-initiated atom transfer radical polymerization using 2-(4-chlorosulfonylphenyl)-ethyltrichlorosilane as an initiator. Based on molecular weight and layer thickness measurements, it was deduced that the apparent graft density was 0.6-0.8 chains $\mathrm{nm}^{-2}$ depending on the polymerization time and that the conformation of tethered chains was highly extended. Surface relaxation behavior of the PMMA brush layer and the spin-coated PMMA film was examined by lateral force microscopy. The $\alpha_{\mathrm{a}}$ - and $\beta$-relaxation processes were discernibly observed at both surfaces. Although surface molecular motion of the brush layer and the spin-coated film was markedly different from the bulk one, both were hardly distinguishable in terms of relaxation phenomena.

KEY WORDS Polymer Brushes / Surface Molecular Motion / Poly(methyl methacrylate)

(PMMA) / Scanning Force Microscopy /
\end{abstract}

Surfaces and interfaces of polymeric materials play an important role in many technological applications such as lubrication, adhesion, biomaterials, etc. ${ }^{1}$ To design highly functionalized polymeric materials, the systematical understanding of aggregation states and physical properties in the vicinity of surface and interfacial layers, which are often quite different from those in the interior bulk region, ${ }^{2}$ is of pivotal importance as the first benchmark.

Thus far we have studied surface molecular motion in polystyrene (PS) films coated onto solid substrates by scanning force microscopy in conjunction with spectroscopy. ${ }^{3}$ Although some groups claimed that there were no any peculiarities for segmental mobility at the surface in PS films, ${ }^{4,5}$ we have definitely observed that glass-rubber transition temperature at the surface, $T_{\mathrm{g}}^{\mathrm{s}}$, was much lower than the corresponding bulk one. ${ }^{3}$ For the moment, such an active thermal molecular motion at the surface has been mainly explained in terms of the following two factors; an excess free volume induced by chain end groups localized at the surface and a reduced cooperativity for $\alpha_{\mathrm{a}}$-relaxation process corresponding to segmental motion at the surface. However, other responsible factors affecting surface molecular motion might be existed. For instance, it seems likely that entanglement density in the vicinity of the surface is markedly reduced in comparison with that in the interior bulk region. ${ }^{6,7}$ Also, chain conformation at the surface would not be the same as that in the bulk phase. ${ }^{8}$ These situations probably make molecular mo- tion active or inactive at the surface. Hence, these effects should be addressed one by one in order to rationalize why peculiar molecular motion manifests at the surface in polymer films.

A highly-distorted chain conformation would be experimentally realized by two ways. One of them is to use ultrathin film ${ }^{9}$ and another is to use polymer brush layer. ${ }^{10}$ A polymer brush layer is composed of tethered chains onto a solid substrate. When an average distance between tethered chains is much smaller than the endto-end distance of an unperturbed chain, the tethered chains are highly stretched out along the direction perpendicular to the surface. Such a well-defined polymer brush layer can be promisingly prepared by a technique of surface-initiating atom transfer radical polymerization (ATRP), ${ }^{11}$ and it has been already elucidated that molecular motion in the brush layer itself is different from that in the bulk. ${ }^{11 \mathrm{~d}}$ Since our interest is strictly the surface, however, thermal molecular motion at the surface of polymer brush layer is explored in this study.

Poly(methyl methacrylate) (PMMA) is used to prepare brush layers because of the following two reasons. First, in the case of PMMA, a preparation route to brush layers has been well established by Yamamoto and coworkers. ${ }^{11}$ Second, we have not systematically studied surface molecular motion of PMMA yet, although it is of importance for optical applications as well as fundamental science. Glass-rubber transition temperature, $T_{\mathrm{g}}$, in PMMA ultrathin films has been studied by ellipsometry, ${ }^{12}$ infrared reflection spectroscopy, ${ }^{13} \mathrm{X}$-Ray

\footnotetext{
$\dagger$ Present address: Institute for Fundamental Research of Organic Chemistry, Kyushu University.

${ }^{\dagger}$ To whom correspondence should be addressed (Fax: +81-92-651-5606, Tel: +81-92-642-3558, E-mail: kajiyama@cstf.kyushu-u.ac.jp).
} 

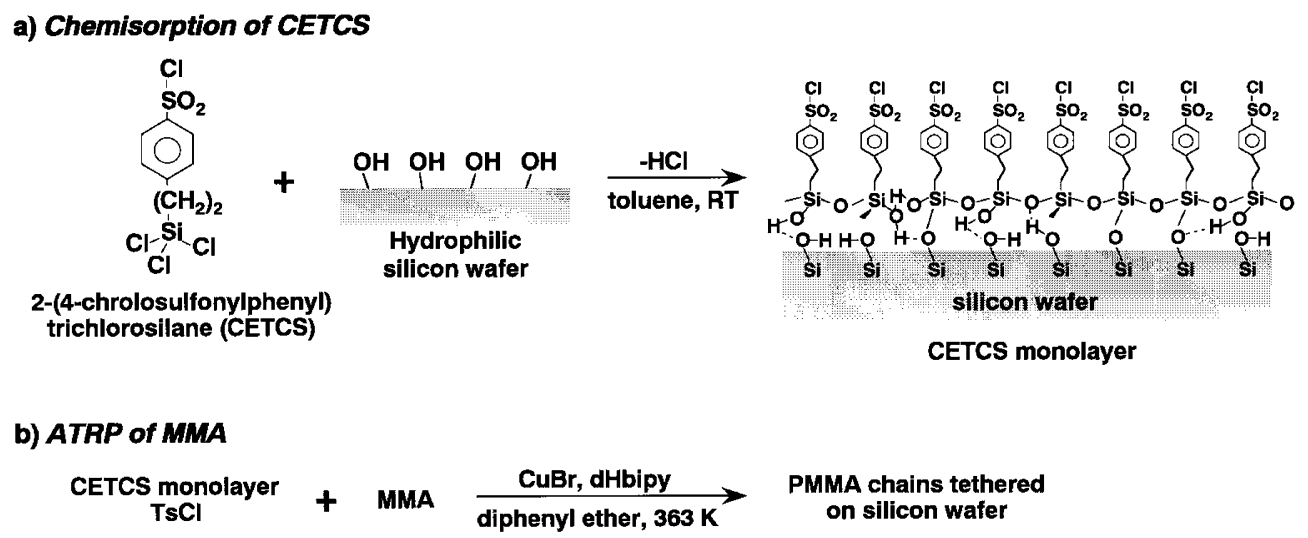

Figure 1. Preparation route of PMMA brushes in this study.

reflectivity ${ }^{14}$ and dielectric relaxation spectroscopy. ${ }^{15}$ Consequently, it was revealed that $T_{\mathrm{g}}$ values in ultrathin films were strongly affected by surface and interfacial layers. In this report, the PMMA brush layer is characterized at first, and then, for a comparison, thermal molecular motion both in the bulk and at the surface of the spin-coated PMMA film is examined. Finally, surface molecular motion of the PMMA brush layers is discussed.

\section{EXPERIMENTAL}

\section{PMMA Brush Preparation}

Well-defined PMMA brush layer was prepared on silicon wafer following the procedure by Yamamoto et al. ${ }^{11}$ A toluene solution of 2-(4-chlorosulfonylphenyl)ethyltrichlorosilane (CETCS, Gelest Co., Ltd.) was prepared with the concentration of $0.5 \mathrm{wt} \%$, and then a hydrophilic silicon wafer was soaked in it for $2 \mathrm{~h}$ at room temperature under nitrogen atmosphere. The obtained CETCS monolayer was used as a surface initiator for the ATRP. In order to carry out graft polymerization of methyl methacrylate (MMA), the silicon wafer covered with CETCS monolayer was immersed at $363 \mathrm{~K}$ for a given time into a degassed diphenyl ether solution containing $\mathrm{CuBr}(10 \mathrm{mM}), 4,4^{\prime}$-di- $n$-heptyl2, 2'-bipyridine (dHbipy, $20 \mathrm{mM}$ ), MMA (4.7 M), and $p$-toluenesulfonyl chloride ( $\mathrm{TsCl}, 3.6 \mathrm{mM})$. TsCl was added as a free initiator not only to control the polymerization but also to produce free polymers, which are effective to characterize the graft chains. ${ }^{11}$ Here, "free" initiator and polymers mean species not immobilized onto the substrate. The obtained PMMA brush layer was washed by Soxhlet extraction using toluene for more than $12 \mathrm{~h}$, and then the brush was dried under vacuum for more than $24 \mathrm{~h}$. The thickness of the PMMA brush layer was determined by ellipsometric measurement (JASCO, M150) using He-Ne laser with the wavelength of $633 \mathrm{~nm}$. Figure 1 shows the prepara- tion route of the PMMA brush layer in this study.

\section{Bulk Characterizations}

The number-average molecular weight, $M_{\mathrm{n}}$, and molecular weight distribution, $M_{\mathrm{w}} / M_{\mathrm{n}}$, where $M_{\mathrm{w}}$ denotes the weight-average molecular weight, of the free PMMA polymer produced in the solution were determined by gel permeation chromatography (GPC) with Shodex KF-804L column and polystyrene standards. Yamamoto et al. confirmed that the $M_{\mathrm{n}}$ and $M_{\mathrm{w}} / M_{\mathrm{n}}$ of the free polymer could be assumed to be the same of those for the brushes under the current polymerization system. ${ }^{11}$ Also, the stereoregularity of the free PMMA was determined by ${ }^{13} \mathrm{C}$-nuclear magnetic resonance $\left({ }^{13} \mathrm{C}\right.$ NMR) spectroscopy, using a JEOL JNMGSX400 NMR spectrometer operating at $400 \mathrm{MHz}$. Bulk glass transition temperature, $T_{\mathrm{g}}^{\mathrm{b}}$, of the free chains was measured by differential scanning calorimetry (DSC8230, Rigaku Co., Ltd.). The specimen was heated up to $423 \mathrm{~K}$ at a rate of $10 \mathrm{~K} \mathrm{~min}^{-1}$ under dried nitrogen purge. The bulk viscoelastic behavior of the free PMMA was measured by Rheovibron (DDV-01FP, Orientec A\&D Co., Ltd.) at $3.5 \mathrm{~Hz}$. Since it was almost impossible to prepare the self-supported film of the free PMMA due to its fragility and small amount, the free PMMA was coated on to a thin polyimide (PI) film with the thickness of $125 \mu \mathrm{m}$. Then, bulk viscoelastic functions of the PMMA coated on PI were measured as a function of temperature.

\section{Surface Characterizations}

Surface relaxation behavior of the PMMA brush layer and the free PMMA spin-coated onto silicon wafer was examined by scanning force microscopy (SPA 300 HV, Seiko Instruments Industry Co., Ltd.) with an SPI 3800 controller. Lateral force microscopic (LFM) measurement was carried out at various temperatures in vacuo so as to avoid the surface oxidation and a capillary force effect induced by surface-adsorbed 


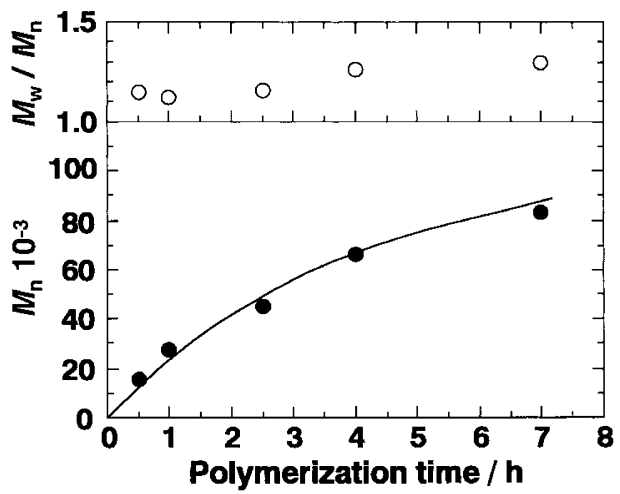

Figure 2. Polymerization time dependence of $M_{\mathrm{n}}$ and $M_{\mathrm{w}} / M_{\mathrm{n}}$ for free PMMA.

water. A piezoscanner was thermally insulated from the heating stage. A cantilever with the bending spring constant of $0.09 \mathrm{Nm}^{-1}$, of which both sides were noncoated, was used. The normal force onto the cantilever was set to be $10 \mathrm{nN}$. To examine surface distribution of chain end groups in the PMMA brush, X-Ray photoelectron spectroscopic (XPS) measurement was carried out. The XPS spectra were obtained with a Phi ESCA 5800 X-Ray photoelectron spectrometer (Physical Electronics Co., Ltd.). The X-Ray source was monochromatic Al- $K_{\alpha}$ operated at $14 \mathrm{kV}$ and $25 \mathrm{~mA}$. The sample stage was kept to be under $173 \mathrm{~K}$ during the measurement.

\section{RESULTS AND DISCUSSION}

Figure 2 shows the polymerization time dependence of $M_{\mathrm{n}}$ and $M_{\mathrm{w}} / M_{\mathrm{n}}$ for free PMMA produced in the solution, in which the surface-initiated ATRP proceeded. $M_{\mathrm{n}}$ monotonically increased with increasing polymerization time, and $M_{\mathrm{w}} / M_{\mathrm{n}}$ was smaller than 1.2 for a shorter polymerization time such as less than $2.5 \mathrm{~h}$. Hence, it seems likely that the polymerization process was well-controlled with a negligible contribution of transfer and termination reactions in this polymerization time. In contrast, in the case of the polymerization for a time longer than $4 \mathrm{~h}, M_{\mathrm{w}} / M_{\mathrm{n}}$ turned to be larger than 1.3. This result implies that the polymerization proceeded in a somewhat uncontrollable manner for such a longer polymerization time in the $\mathrm{Cu}(\mathrm{I}) \mathrm{Br} / \mathrm{dHbipy}$ system.

Figure 3 shows the layer thickness of the PMMA brush prepared by ATRP for 5 different times. Assuming that $M_{\mathrm{n}}$ of the free PMMA is the same as that of the PMMA brush, ${ }^{11}$ the abscissa of Figure 3 is expressed by the corresponding $M_{\mathrm{n}}$ of the free PMMA to each polymerization time. The predicted relationships between layer thickness and $M_{\mathrm{n}}$ for the PMMA with all-trans and random coil conformations are also

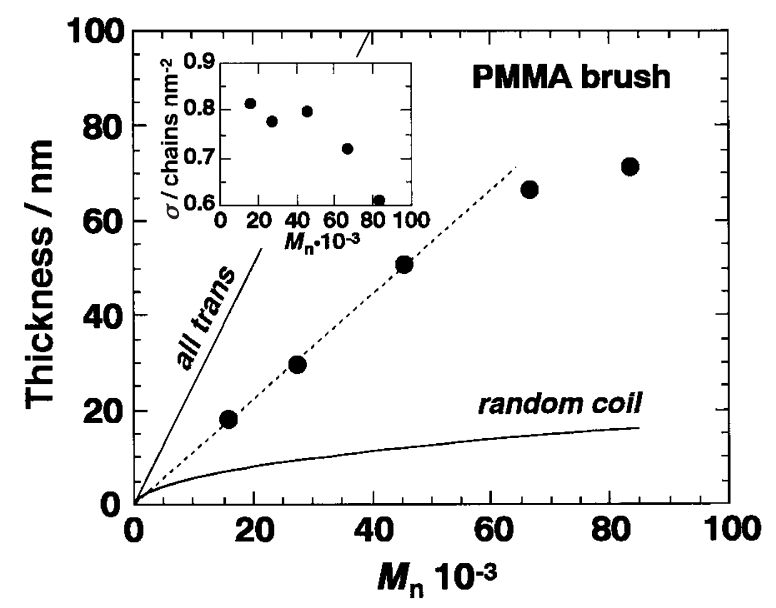

Figure 3. Relationship between $M_{\mathrm{n}}$ and layer thickness for the obtained PMMA brush layer, provided that brush's $M_{\mathrm{n}}$ is assumed to be the same as that of the free chains. The solid line and curve are drawn in the context of all-trans and random coil conformations, respectively. The inset shows the apparent graft density variation with $M_{\mathrm{n}}$.

depicted in Figure 3 by solid lines. Both lines were given by $0.254 \mathrm{~N}$ and $2\left(\mathrm{Nb}^{2} / 6\right)^{1 / 2}$, respectively, where $N$ and $b$ are the degree of polymerization and the statistical segment length of $0.68 \mathrm{~nm}$. The layer thickness of the brush obtained was intermediate value between the two limits, namely, all-trans and random coil conformations, at a given $M_{\mathrm{n}}$. Hence, it is conceivable that the tethered PMMA chains have an extended conformation along the direction normal to the surface. The layer thickness of the brush prepared by ATRP linearly increased up to $M_{\mathrm{n}}=45.4 \mathrm{k}$, and then the relation was apparently deviated from the linearity. Apparent graft density of the PMMA brushes calculated by layer thickness, $M_{\mathrm{n}}$ and the assumed density of $1.19 \mathrm{~g} \mathrm{~cm}^{-3}$ was shown as a function of $M_{\mathrm{n}}$ in the inset of Figure 3. Although the apparent graft density was kept to be approximately 0.8 chains $\mathrm{nm}^{-2}$ up to $M_{\mathrm{n}}=45.4 \mathrm{k}$, those values for the PMMA brushes with $M_{\mathrm{n}}$ of $66.5 \mathrm{k}$ and $83.3 \mathrm{k}$ were 0.72 and 0.61 , respectively. These results indicate that in the case of the long brushes with $M_{\mathrm{n}}$ of $66.5 \mathrm{k}$ and $83.3 \mathrm{k}$, the polymerization was not necessarily controlled as desired, and are in good accordance with the conclusion made after Figure 2. Here, it should be noted that $M_{\mathrm{n}}$ value of the tethered PMMA was assumed to be the same as that for the corresponding free chains, as mentioned above. If this assumption is not the case in reality, especially for a longer time, the aforementioned notion related to controllability of the surface-initiated ATRP should not be claimed for the moment. To conclude this issue, further study should be somehow made. In any event, however, it seems reasonable to consider that the tethered PMMA with $M_{\mathrm{n}}$ of $45.4 \mathrm{k}$, or smaller, are well-defined. Hence, 


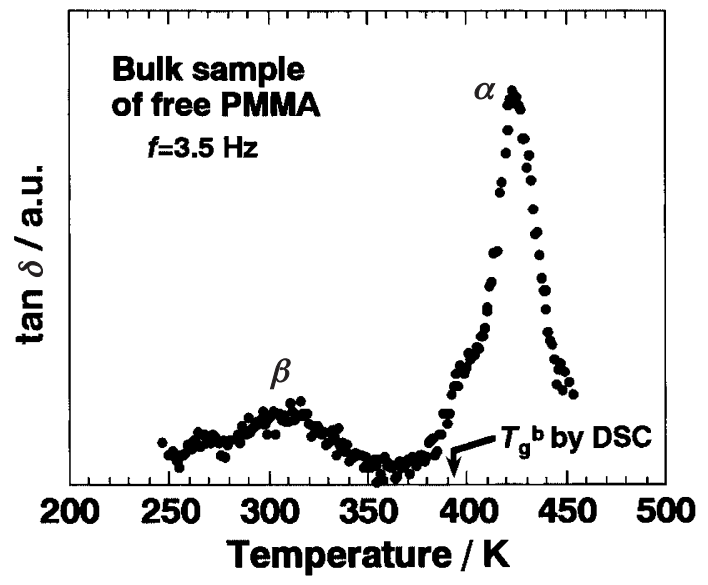

Figure 4. Temperature dependence of dynamic loss tangent for the bulk sample of free PMMA. The measuring frequency was $3.5 \mathrm{~Hz}$. The arrow denotes bulk $T_{\mathrm{g}}$ measured by DSC.

the following study was carried out using the free and tethered PMMA with $M_{\mathrm{n}}$ of $45.4 \mathrm{k}$.

Before starting discussion about thermal molecular motion of the PMMA, the stereoregularity of the PMMA should be addressed because they generally have a strong correlation. ${ }^{13,16}$ Based on diad by ${ }^{13} \mathrm{C}$ NMR measurement, the racemo fraction of the PMMA was 0.57 , indicating that the PMMA used was syndiotactic rich. Figure 4 shows the temperature dependence of $\tan \delta$ for the bulk PMMA with $M_{\mathrm{n}}$ of $45.4 \mathrm{k}$, which obtained in the solution, at the measuring frequency of $3.5 \mathrm{~Hz}$. In this figure, two clear peaks were observed. The broad peak at the lower temperature side has been hitherto understood as $\beta$-relaxation process arising from the hindered rotation of the -COO$\mathrm{CH}_{3}$ around the $\mathrm{C}-\mathrm{C}$ bond which links it to the main chain part. ${ }^{16,17}$ On the other hand, the peak observed at the higher temperature side can be assigned to $\alpha_{\mathrm{a}}$ absorption corresponding to micro-Brownian motion of the main chain. The onset temperature of the $\alpha_{\mathrm{a}}$ absorption peak was in good accordance with $T_{\mathrm{g}} \mathrm{b}$ measured by DSC.

Since the manifestation of frictional force of polymeric solids is closely related to their viscoelastic properties, ${ }^{3 a, 3 b, 4,18}$ it is possible to examine surface molecular motion of the polymeric solids by using LFM. When the energy dissipation increases at the surface due to molecular motion, lateral force increases. Hence, it can be postulated that lateral force alteration with measuring temperature is essentially similar to the temperature dependence of dynamic loss modulus or mechanical loss tangent. ${ }^{3,4,18}$ Figure 5 shows the lateral force $v s$. temperature curves for (a) the spincoated PMMA film and (b) the PMMA brush layer at the fixed scanning rate of $10^{3} \mathrm{~nm} \mathrm{~s}^{-1}$. The scanning rate of $10^{3} \mathrm{~nm} \mathrm{~s}^{-1}$ for LFM can be converted to

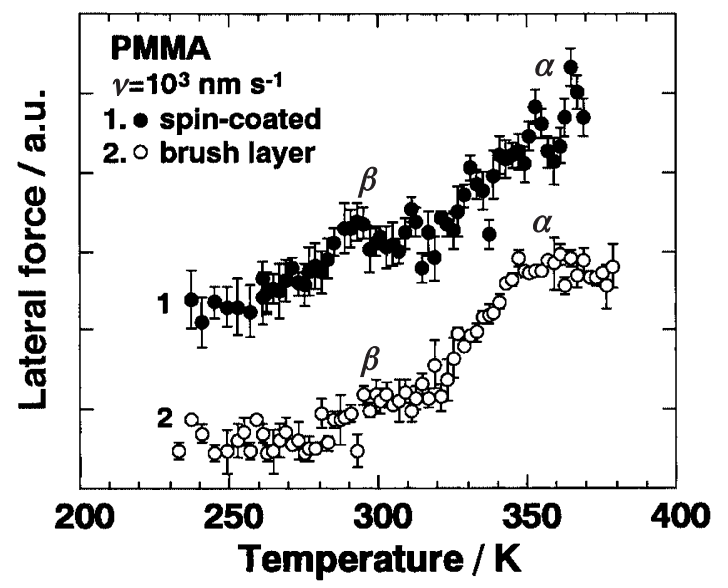

Figure 5. Typical lateral force-temperature curves for the spincoated film and the brush layer of PMMA at the scanning rate of $10^{3} \mathrm{~nm} \mathrm{~s}^{-1}$.

the frequency of about $70 \mathrm{~Hz}$ on the basis of JohnsonKendall-Roberts theory. ${ }^{19}$ Hence, if the data shown in Figure 5 is acquired at the scanning rate corresponding to the frequency of $3.5 \mathrm{~Hz}$, the peak positions will be shifted to a lower temperature side. The PMMA used for the spin-coated film was obtained in the same solution, in which the brush layer was prepared by the surface-initiated ATRP. Thus, both the spin-coated film and the brush layer possessed the same primary structure, ${ }^{11}$ and its $M_{\mathrm{n}}$ was $45.4 \mathrm{k}$. The thickness of the spin-coated film was $58 \mathrm{~nm}$ being comparable to the brush layer thickness of $52 \mathrm{~nm}$. In the case of $T_{\mathrm{g}}$ in thin PMMA films, $T_{\mathrm{g}}$ value would be dependent on the thickness, ${ }^{12-15}$ whereas surface segmental mobility was not the case in this thickness range for the current experimental condition. As shown in Figure $5, \alpha_{\mathrm{a}}$-relaxation process was clearly observed accompanying a small peak or shoulder at both sample surfaces. Although the small peak or shoulder is not clear in comparison with the $\alpha_{\mathrm{a}}$-absorption peak, we assigned it to a surface $\beta$-process owing to the following four reasons; (1) this slight peak or shoulder was not observed for the case of PS, (2) the reproducibility was quite good, (3) the $\beta$-process was observed for bulk PMMA sample as well, as shown in Figure 4, and (4) the $\beta$-process was appeared even in a confined system such as ultrathin films. ${ }^{15}$ In general, it has been well accepted that molecular motion of side chain groups, namely, $\beta$-relaxation, is independent of their circumstances or aggregation states. ${ }^{16}$ Nevertheless, the surface $\beta$-relaxation peak for the spin-coated film and the brush layer was observed at a temperature lower than that for the bulk sample by $20 \mathrm{~K}$. Similarly, it has been observed that the $\beta$-relaxation temperature for ultrathin PMMA films decreases with decreasing film thickness. ${ }^{15}$ Hence, it is plausible that the $\beta$-relaxation 
of PMMA is a little complicated process composed of the hindered rotation of side chain groups and some sort of the local cooperative movement. Similar discussion of the coupling between $\beta$ - and $\alpha_{\mathrm{a}}$-relaxation processes for bulk PMMA was made by Spiess et al. using multidimensional NMR measurements. ${ }^{20}$ The second distinct increase in lateral force can be assigned to the surface $\alpha_{\mathrm{a}}$-relaxation corresponding to micro-Brownian motion of the main chain part, and was observed at a temperature much lower than $T_{\mathrm{g}}^{\mathrm{b}}$ of $393 \mathrm{~K}$, meaning that molecular motion at the surface is definitely activated in comparison with that in the internal bulk region. Since the surface $\beta$ - and $\alpha_{\mathrm{a}}$-relaxation peaks were in part overlapped, the precise value of $T_{\mathrm{g}} \mathrm{s}$ can be hardly determined. While in the case of the brush layer, the peak top for the surface $\alpha_{\mathrm{a}}$-relaxation was clearly observed, this was not the case for the spin-coated film at the temperature range employed. If the peak width, namely, the distribution of relaxation time, is the same in the two, $T_{\mathrm{g}} \mathrm{s}$ of the brush layer can be claimed to be lower than that of the spin-coated film based on the relation of the peak positions for the surface $\alpha_{\mathrm{a}}$-relaxation. However, such a speculation is somewhat dangerous at present. Thus, it seems a little early to conclude from Figure 5 that thermal molecular motion at the surface of the brush layer differs from that of the spin-coated film.

Finally, it was examined by XPS whether end groups of the tethered chains still remained at the top surface of the brush layer even after a successive drying process to ATRP. The end portion of a tethered chain is supposed to be terminated by chlorine. Thus, it has a relative high polarity in comparison with the main chain part. On the XPS $\mathrm{Cl}_{2 \mathrm{p}}$ spectrum for the brush layer, a discernible peak was not observed even at the emission angle of photoelectrons of $15^{\circ}$. This result makes it clear that the chain end portion of the tethered chains deeply migrated into the interior bulk region over the analytical depth of the current XPS measurement, approximately $2.7 \mathrm{~nm}$. To realize such a situation, it is conceivable that chain conformation in the surface region of the brush layer is highly randomized. In addition, the tethered chains have various lengths, as predicted from the $M_{\mathrm{w}} / M_{\mathrm{n}}$ value of 1.2 , and thus, the actual graft density in the vicinity of the surface would be much smaller than that in the interior region. This results in that chain conformation in the surface region of the brush layer is no longer extended unlike its interior bulk region. If that is the case, it seems most likely that the surface of the brush layer is not so different from the spin-coated one in terms of the chain conformation. Since the indentation depth of an LFM probe tip can be estimated to be less than $1 \mathrm{~nm},{ }^{3 \mathrm{~h}}$ relaxation behavior at the surface of the brush layer could be hardly distinguished from that in the corresponding spin-coated film.

\section{CONCLUSIONS}

Well-defined PMMA brush layers were prepared onto silicon wafers by surface-initiated ATRP and its surface relaxation behavior was studied by LFM. Although surface molecular motion of the brush layer was quite different from the bulk one, a clear discrepancy of surface molecular motion between the brush layer and the spin-coated film could be hardly observed.

Acknowledgment. We are most grateful for fruitful discussions about surface-initiated ATRP with Prof. Takeshi Fukuda, Prof. Yoshinobu Tsujii, and Dr. Shinpei Yamamoto, Kyoto University, and Prof. Hideyuki Otsuka, Kyushu University. This was in part supported by a Grant-in-Aid for Scientific Research (A) (No.13355034) from the Ministry of Education, Culture, Sports, Science and Technology, Japan, and by the Foundation "Hattori-Hokokai".

\section{REFERENCES}

1. F. Garbassi, M. Morra, and E. Occhiello, "Polymer Surfaces, from Physics to Technology”, John Wiley \& Sons Ltd., Chichester, 1994.

2. R. A. L. Jones and R. W. Richards, "Polymers at Surfaces and Interfaces", Cambridge University Press, Cambridge, 1999.

3. a) T. Kajiyama, K. Tanaka, I. Ohki, S.-R. Ge, J.-S. Yoon, and A. Takahara, Macromolecules, 27, 7932 (1994).

b) T. Kajiyama, K. Tanaka, and A. Takahara, Macromolecules, 30, 280 (1997).

c) K. Tanaka, A. Takahara, and T. Kajiyama, Macromolecules, 30, 6626 (1997).

d) K. Tanaka, A. Takahara, and T. Kajiyama, Macromolecules, 31, 863 (1998).

e) K. Tanaka, X. Jiang, K. Nakamura, A. Takahara, T. Kajiyama, T. Ishizone, A. Hirao, and S. Nakahama, Macromolecules, 31, 5148 (1998).

f) T. Kajiyama, K. Tanaka, N. Satomi, and A. Takahara, Macromolecules, 31, 5150 (1998).

g) N. Satomi, A. Takahara, and T. Kajiyama, Macromolecules, 32, 4474 (1999).

h) K. Tanaka, A. Takahara, and T. Kajiyama, Macromolecules, 33, 7588 (2000).

i) N. Satomi, K. Tanaka, A. Takahara, and T. Kajiyama, Macromolecules, 34, 6420 (2001).

j) D. Kawaguchi, K. Tanaka, A. Takahara, and T. Kajiyama, Macromolecules, 34, 6164 (2001).

k) N. Satomi, K. Tanaka, A. Takahara, and T. Kajiyama, Macromolecules, 34, 8761 (2001).

1) F. Xie, H. F. Zhang, F. K. Lee, O. K. C. Tsui, Y. Yokoe, K. Tanaka, A. Takahara, T. Kajiyama, and T. He, Macro- 
molecules, 35, 1491 (2002).

m) K. Tanaka, T. Kajiyama, A. Takahara, and S. Tasaki, Macromolecules, 35, 4702 (2002).

4. F. Dinelli, C. Buenviaje, and R. M. Overney, J. Chem. Phys., 113, 2043 (2000).

5. S. Ge, Y. Pu, W. Zhang, M. Rafailovich, J. Sokolov, C. Buenviaje, R. Buckmaster, and R. M. Overney, Phys. Rev. Lett., 85, 2340 (2000).

6. H. R. Brown and T. P. Russell, Macromolecules, 29, 798 (1996).

7. Y. C. Jean, R. Zhang, H. Cao, J.-P. Yuan, C.-M. Huang, B. Nielsen, and P. Asoka-Kumar, Phys. Rev. B, 56, R8459 (1997).

8. I. A. Bitsanis and G. ten Brinke, J. Chem. Phys., 99, 3100 (1993).

9. K. Akabori, K. Tanaka, A. Takahara, and T. Kajiyama, in preparation.

10. In this paper, we define polymer brushes as tethered polymer chains in a dried state.

11. a) E. Muhammad, S. Yamamoto, K. Ohno, Y. Tsujii, and T. Fukuda, Macromolecules, 31, 5934 (1998).

b) S. Yamamoto, E. Muhammad, Y. Tsujii, M. Matsumoto, and T. Fukuda, Macromolecules, 33, 5602 (2000).

c) S. Yamamoto, E. Muhammad, Y. Tsujii, and T. Fukuda,
Macromolecules, 33, 5608 (2000).

d) S. Yamamoto, Y. Tsujii, and T. Fukuda, Macromolecules, 35, 6077 (2002).

12. J. L. Keddie, R. A. L. Jones, and R. A. Cory, Faraday Discuss., 98, 219 (1994).

13. Y. Grohens, M. Brogly, C. Labbe, M. O. David, and J. Schultz, Langmuir, 14, 2929 (1998).

14. D. S. Fryer, R. D. Peters, E. J. Kim, J. E. Tomaszewski, J. J. de Pablo, P. F. Nealey, C. C. White, and W. L. Wu, Macromolecules, 34, 5627 (2001).

15. K. Fukao, S. Uno, Y. Miyamoto, A. Hoshino, and H. Miyaji, Phys. Rev. E: Stat Phys., Plasmas Fluids, Relat. Interdiscip. Tap, 64, 51807 (2001).

16. N. G. McCrum, B. E. Read, and G. Williams, "Anelastic and Dielectric Effects in Polymeric Solids", Dover, New York, N.Y., 1967.

17. J. A. Prins, "Physics of Non-Crystalline Solids", North Holland, Amsterdam, 1965.

18. J. A. Hammerschmidt, W. L. Gladfelter, and G. Haugstad, Macromolecules, 32, 3360 (1999).

19. K. L. Johnson, K. Kendall, and A. D. Roberts, Proc. R. Soc. London A, 324, 301 (1971).

20. S. C. Kuebler, D. J. Schaefer, C. Boeffel, U. Pawelzik, and H. W. Spiess, Macromolecules, 30, 6597 (1997). 